Lars Olof Larsson

\title{
„DIE VORNEHMSTEN PLÄTZE UND GEBÄUDE, DIE IN DANZIG ZU SEHEN SIND“. AEGIDIUS DICKMANS ANSICHTEN VON DANZIG 1617
}

1617 publizierte der sonst wenig bekannte Aegidius Dickman ein großes Stadtpanorama von Danzig und eine Folge von Ansichten verschiedener Gebäude und Plätze der Stadt. Es handelt sich um graphische Blätter, überwiegend in Radiertechnik ausgeführt. In der Signatur auf dem Panorama gibt Dickman an, aus Danzig zu stammen und die Drucke selbst zu verlegen: Ægidius Dickman Gedanensis fecit et excudebat 1617 lautet die Signatur. Wie es scheint, handelte er auf eigene Initiative und nicht im Auftrag etwa des Rats. Vom Stadtpanorama, das mit einer Länge von $258 \mathrm{~cm}$ und einer Höhe von $53,7 \mathrm{~cm}$ fast alle bekannten graphischen Stadtveduten größenmäßig übertrifft, ist nur ein einziges Exemplar bekannt, und die Folge der Stadtansichten ist ebenfalls selten. ${ }^{1}$ Wie groß die ursprünglichen

DOI: https://doi.org/10.12697/BJAH.2020.20.03

1 Das Panorama befindet sich in der Königlichen Bibliothek in Stockholm: Isak Collijn, „Magnus Gabriel de la Gardies samling af äldre stadsvyer och historiska planscher i Kungl. Biblioteket”, Kungl. bibliotekets handlingar 35. Årsberättelse för år 1912 (Stockholm, 1915), 57f., Nr. 163, Tafel 12; Hollstein's German Etchings, Engravings and Woodcuts c. 1400-1700, vol. 6 (1960), Nr. 1. Sieben Blätter der Stadtansichten von 1617 befinden sich im Germanischen Nationalmuseum [GNM] in Nürnberg. Sie sind hier abgebildet. Vgl. Von Danzig bis Riga. Ansichten, Stadtpläne und Landkarten von Ost-und Westpreußen, Danzig und dem Baltikum aus der graphischen Sammlung des Germanischen Nationalmuseums (Nürnberg: Germanisches Nationalmuseum Nürnberg, 1982), Nr. 20-29. Egidius Dickmann und das Danziger Stadtbild um 1617, mit Einführung und Kommentaren von Ernst Bahr, hrsg. von Eckahrd Jäger (Lüneburg: Nordostdt. Kulturwerk, 1979), bildet alle Radierungen nach der von Claus Jansz. Visscher 1625 herausgegeben Folge ab. Die in dieser Publikation enthaltenen Zusammenfassung der bekannten Daten zur Person Dickmans und Bildkommentare bilden die Grundlage für jede weitere Beschäftigung mit Dickmans Werk. 
Auflagen waren, wissen wir natürlich nicht. Sie müssen aber rasch Verbreitung gefunden haben, denn eine Kopie der Vedute mit Motivanleihen vom Titelblatt der Folge der Stadtansichten beweisen, dass sie schon ein Jahr nach ihrem Erscheinen in Amsterdam bekannt waren. Aus der Sicht Dickmans gehörten Panorama und Stadtansichten sicher insofern zusammen, als sie sich inhaltlich ergänzten. Schon die unterschiedlichen Formate zeigen aber, dass sie als selbständige, voneinander unabhängige Produkte auf den Markt gebracht wurden. Im Folgenden soll es darum gehen, den Rang der Stadtansichten im Kontext der topographischen Druckgraphik der Zeit um $1600 \mathrm{zu}$ untersuchen und ihren künstlerischen Charakter zu erläutern. ${ }^{2}$ Ihr dokumentarischer Wert in Bezug auf die Topographie und Baugeschichte Danzigs wurde bereits von Ernst Bahr ausführlich gewürdigt und soll hier nur am Rande berücksichtigt werden.

Die Folge setzt sich zusammen aus einem Titelblatt, zwölf Ansichten von verschiedenen Örtlichkeiten der Stadt und einem Stadtplan. Die Blätter messen je etwa $17 \times 30 \mathrm{~cm}$. Vermutlich wurden sie als lose Bögen verkauft, die der Käufer selbst zu einem Heft zusammenbinden sollte. Dafür, dass sie zu einem Heft vereint werden sollten, spricht das repräsentative Titelblatt sowie die Tatsache, dass die Blätter in der 1625 bei Claes Jansz. Visscher in Amsterdam erschienenen zweiten Auflage nummeriert sind. Auf die Frage, ob die damit angegebene Reihenfolge einer besonderen Systematik folgt, werden wir zurückkommen.

Wie es zur Neuauflage der Drucke kam, wissen wir nicht. Man kann aber vermuten, dass Dickman nach Holland gereist ist, nachdem er erfahren hatte, dass der Amsterdamer Verleger Pieter van der Keere eine Kopie seines großen Stadtpanoramas herausgegeben hatte. ${ }^{3}$ Im Frühjahr 1624 ist Dickmans Anwesenheit in Haarlem dokumentiert. Um sich vor weiteren Verlusten zu schützen, kann er sich mit dem Verleger Claes Jansz. Visscher in Verbindung gesetzt und eine Zusammenarbeit eingeleitet haben. Vielleicht bestand auch schon vorher eine Verbindung mit Visscher; vieles

2 Zum großen Panorama siehe Egidius Dickmann und das Danziger Stadtbild um 1617, 29-42, und Lars Olof Larsson, „DANTISCUM SEPTENTRIONIS, OCCIDENTIS ET TOTIUS ORBIS
FREQUENTISSIMUM HODIE EMPORIUM. Ægidius Dickmans große Panorama von Danzi FREQUENTISSIMUM HODIE EMPORIUM. Ægidius Dickmans große Panorama von Danzig
von $1617^{\circ}$, Porta aurea, 19 (2020). Jubiläumsband anlässlich des 60 . Geburtstages von Professo Malgorzata Omilanowska, hrsg. von D. Konstantynów und A. Sobecka, 77-95.

3 Egidius Dickmann und das Danziger Stadtbild um 1617, 12; Larsson, „DANTISCUM SEPTENTRIONIS, OCCIDENTIS ET...“, 80-83, 91 . spricht dafür, dass Dickman in Holland ausgebildet worden war. Auf dem Titelblatt der neuen Auflage ist links unten am Postament Dickmans Signatur und die Datierung $1625 \mathrm{zu}$ lesen: $Æ D$ fec. Anno 1625. Eine Leerstelle zwischen $\mathrm{fec}$. und der Jahreszahl zeigt, dass die Originalplatte wiederverwendet wurde, nachdem die Verlegerangabe (et exc.) ausradiert und die Jahreszahl geändert worden waren. Am Sockel rechts unten hat Visscher seine Verlegersignatur C.J.Visscher excudebat hinzugefügt. Auf den folgenden Blättern fehlt, wie auf den Blättern der Originalausgabe auch, die Signatur Dickmans, alle zeigen aber Visschers Monogramm mit oder ohne den Zusatz exc. Visscher dürfte die Druckplatten von Dickman erworben haben und seine Erben haben sie dann später vermutlich weiterverkauft. 1670 ist bei dem Amsterdamer Verleger Frederick de Wit eine dritte Auflage der Folge erschienen, immer noch mit der Signatur Dickmans und der Jahreszahl 1625 auf dem Titelblatt, aber jetzt natürlich mit der Verlegersignatur de Wits.

Der Titel der Folge lautet: PRÆCIPIOR. LOCORUM ET ÆDIFICIORUM QUÆ IN URBE DANTISCANA VISUNTUR ADUMBRACIO (Darstellung der vornehmsten Plätze und Gebäude, die in Danzig zu sehen sind).

Das Titelblatt zeigt einen breiten Torbogen mit einem vorspringenden Proszenium (Abb. 1). Durch die Bogenöffnung sieht man die Stadt wie auf einer Bühne präsentiert, hinter ihr ahnt man die Weichsel und am Horizont die Ostsee. Die Ansicht ist vom Hagelsberg aus, also vom Nordwesten und nicht, wie Dickmans große Stadtpanorama, vom weiter südlich gelegenen Bischofsberg aus gezeichnet worden. In der Bogenöffnung, zwischen Girlanden befestigt, hängt eine Kartusche mit der Titelinschrift. Vergleicht man das Panorama der Stadt mit der Darstellung in Braun und Hogenbergs Civitates Orbis Terrarum (Abb. 3), erkennt man, dass Dickman seine Vedute zwar etwa von der gleichen Stelle aus aufgenommen hat wie der Zeichner der älteren Ansicht, dass es sich aber nicht um eine Kopie des bekannten Vorgängerdruckes handelt. Dickman wählte einen etwas nördlicher gelegenen Standpunkt und konnte daher die Stadttore und Wälle im Norden der Stadt besser darstellen. Sein Panorama macht auch den Eindruck, nur das zu zeigen, was von einem Aussichtspunkt auf dem Hagelsberg tatsächlich zu sehen war; die flache Landschaft des Weichseldeltas wird bei ihm weitgehend von der Stadt verdeckt, während die Darstellung von Braun und 


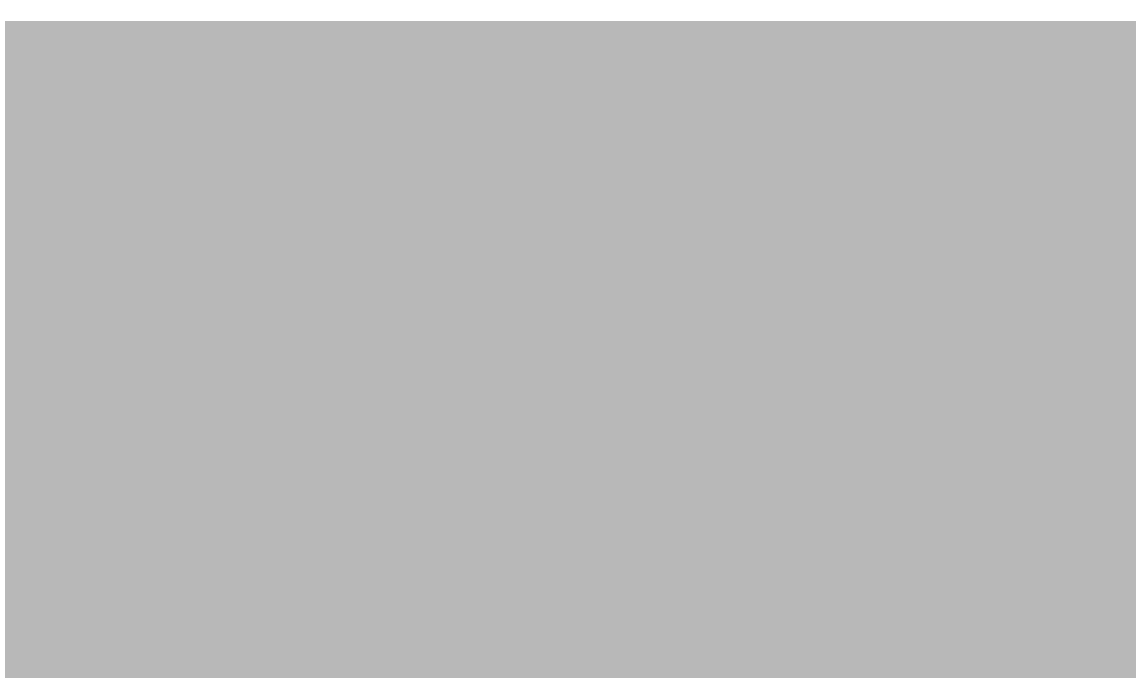

ABB. 1. ÆGIDIUS DICKMANN UND DAS DANZIGER STADTBILD UM 1617, TITELBLATT (1617). FOTO: GNM.

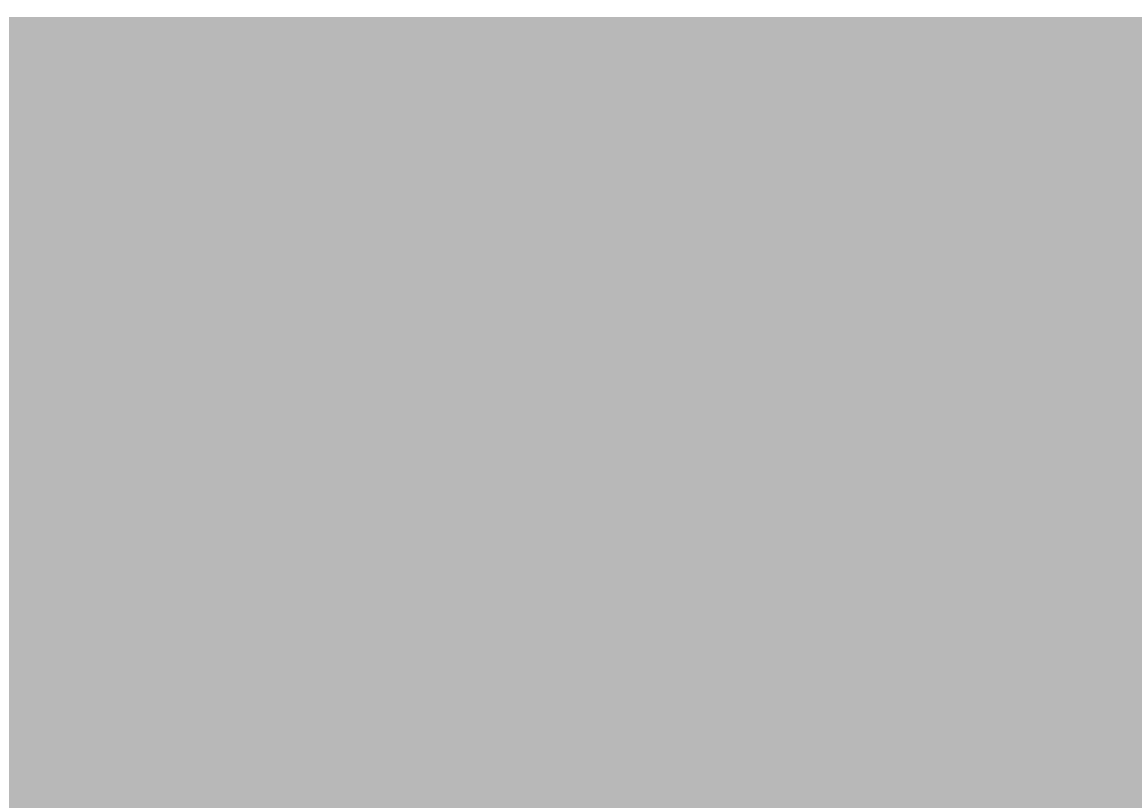

ABB. 2. CLAES JANSZ. VISSCHER, TITELBLATT VON PLAISANTE PLAETSEN... (1612).

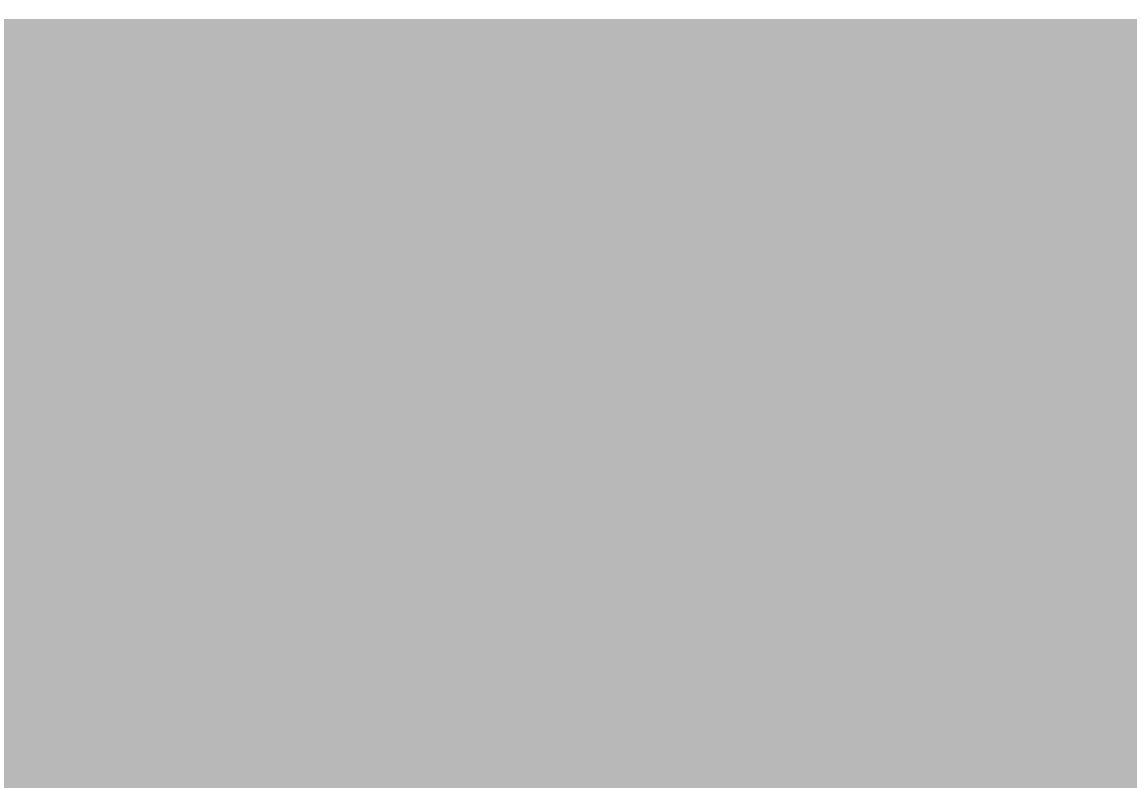

ABB. 3. BRAUN UND HOGENBERG, ANSICHT VON DANZIG, AUS: CIVITATES ORBIS TERRARUM (1575).

Hogenberg die umgebende Landschaft aus der Vogelperspektive umfassend wiedergibt.

Vor dem Bogen stehen links ein älteres und rechts ein jüngeres Paar - beide vornehm, aber sehr verschieden gekleidet. Trägt das ältere Paar die Kleidung würdiger Patrizier, tritt das jüngere Paar mit modischer Eleganz auf. Die beiden Paare lassen an solche Figuren denken, die man z. B. im Vordergrund auf vielen Stadtansichten von Braun und Hogenberg findet. Sie zeigen die Bürger der jeweiligen Stadt in ihrer charakteristischen Tracht. Das dürfte auch hier die Absicht sein. Dabei ist freilich nicht zu übersehen, dass das junge Paar, was ihre Kleidung und ihren Habitus betrifft, kaum spezifisch ortstypische Elemente zeigt und ebenso gut aus einer der beliebten „Fröhlichen Gesellschaften“ des niederländischen Künstlers Willem Buytewech (1591-1624) hätten stammen können. Der Versuch, die soziale Zugehörigkeit der Frauen mit Hilfe von Anton Möllers Frauentrachtenbuch von 1601 etwas genauer zu bestimmen, führt 


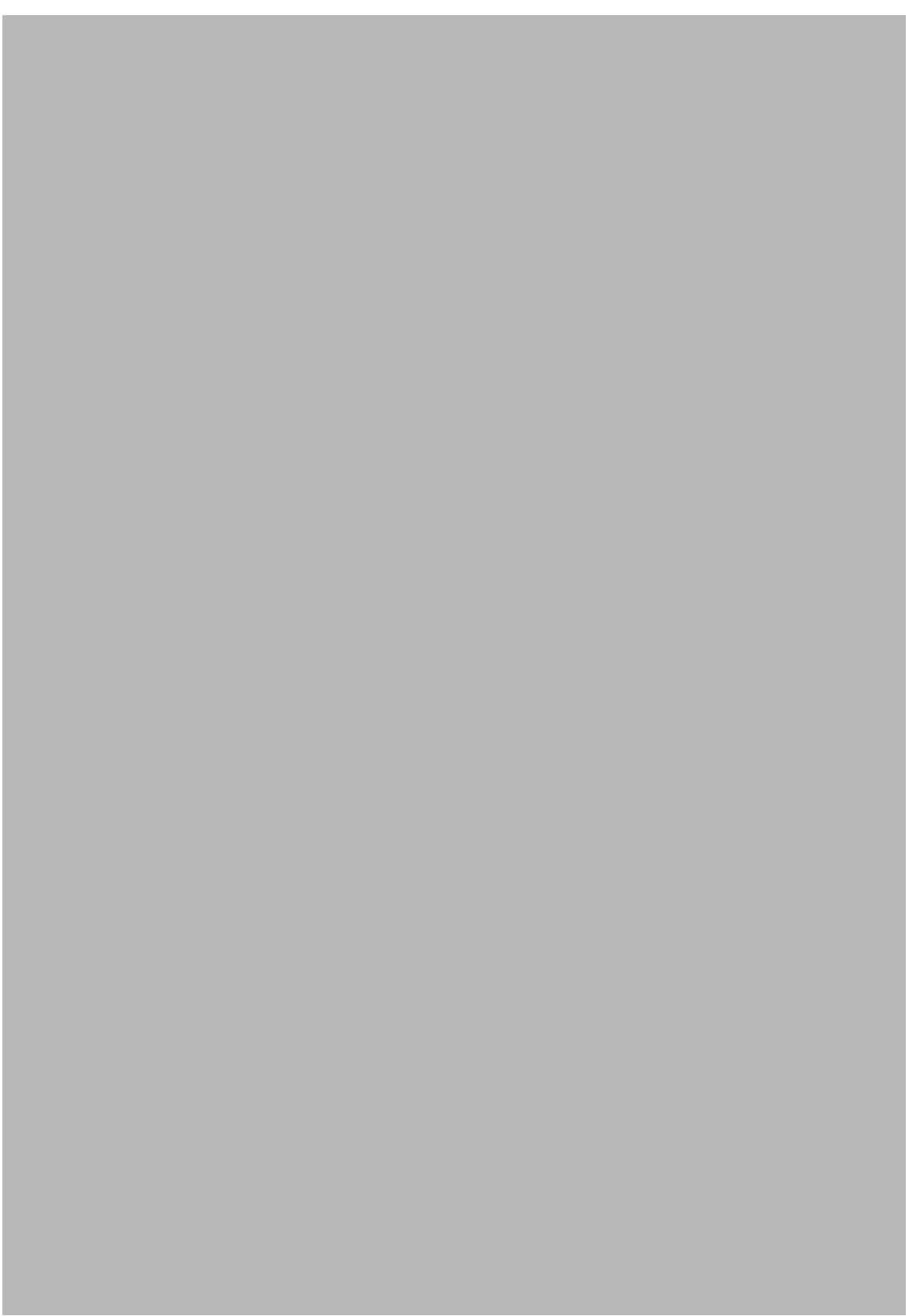

ABB. 4. ANTON MÖLLER, MATRONÆ EXSPANTIANTES. AUS: DER DANTZIGER FRAWEN UND JUNGFRAWEN GEBREUCHLICHE ZIERHEIT UND TRACHT... (1601). leider auch nicht weit. Die ältere Frau ist am ehesten mit Möllers Bild von Spazirenden Frauwen (Matronæ exspatiantes) zu vergleichen (Abb. 4), von denen es heißt:

„Auff'den Newgarten wolln wir gehn / und sehn wie unser rößlein stehn. Die Rosmarien und Kreutlein fassn / Salat und Kreß heim tragen lassn. ${ }^{44}$ Das Paar auf dem Titelblatt gehörte ja auch sicher zu der wohlhabenden Schicht der Danziger Bürger, die vor der Stadt Land besaß und dort auch eigene Pferde hielt.

An den Seiten des Proszeniums sind unter dem älteren Paar Darstellungen zu sehen, die auf den gewinnbringenden Handel anspielen: Schiffe, Warenballen und eine von Pferden gezogene, schwer beladene Fuhre. Unter dem jüngeren Paar sehen wir eine Jagdszene; was die Szene auf der beschatteten, nach Innen gewandten Seite darstellt, erkennt man leider nicht. Die Jagdszene unterstreicht den Status der Danziger Patrizier. Vielen von ihnen gehörten nicht nur Gärten vor der Stadt, sie besaßen auch feudale Güter. Damit verbunden waren auch das Jagdrecht und ein soziales Prestige, das den Besitzer auf eine Stufe mit dem Adel stellte, und sie haben dementsprechend auch einen adeligen Lebensstil gepflegt. ${ }^{5}$ Dass die Jagdszene dem jüngeren Paar zugeordnet ist, will wohl vor allem zeigen, dass die Jagd eine Beschäftigung der Jüngeren war, es mag aber vielleicht auch ein Hinweis darauf sein, dass der damit verbundene herrschaftliche Lebensstil ein neues Phänomen in der Danziger Bürgerschaft sei. In einem anderen Zusammenhang hätte man in dieser Gegenüberstellung einer der ernsten Arbeit verpflichteten älteren und einer dem Vergnügen zugewandten jüngeren Generation eine moralisierende Absicht vermuten können. Das verbietet sich allerdings hier, war es doch sicher das Anliegen der Bilderfolge, die Stadt und ihre Bewohner in positivem Licht darzustellen.

Im Vordergrund liegen ein überquellendes Füllhorn, ein Medusenhaupt und ein Spiegel. Wie dieses emblematisch anmutende Stillleben zu verstehen ist, erläutern lateinische Distichen am Postament:

4 Anton Moeller's Danziger Frauentrachtenbuch aus dem Jahre 1601 in getreuen FaksimileReproduktionen neu herausgegeben nach den Original-Holzschnitten, hrsg von August Bentling (Danzig, 1886). Der begrenzte dokumentarische Wert der Trachtenbücher geht aus Rolf Walthers Untersuchung von Möllers Werk (Rolf Walther, Das Danziger Frauentrachtenbuch von Anton Möller und seine Vorläufer im 16. Jahrhundert, hrsg. von Ernst Bahr (Marburg, 1963), 447469) deutlich hervor.

5 Peter Oliver Loew, Danzig. Biographie einer Stadt (München: Beck, 2011), 86. 
De locuplete penu multis alimenta ministras Terris, hinc CERERIS diceris AULA Deae Ac merito, tibi nam fundit sua munera cornu Divite, Diva CERES; divitasque ferens.

Non fex illa tibi, cui non vexilla politae

Palladis arrident, desidiosa placet.

Barbariem jugulus: stricto petis ense MEDUSAM

Dum Phoebum in gremio nidificare iuvas.

(Aus übervollem Speicher reichst Du von hier / vielen Ländern die Früchte der Ceres: Du Scheuer der Göttin. / Und mit Recht: denn Dir spendet sie Reichtum bringend ihre Gaben aus vollem Horn: die göttliche Ceres.

Nicht gefällt jener träge Pöbel Dir / dem die Feldzeichen der schönen Pallas nicht lächeln. / Du vernichtest die Barbarei, mit starkem Schwert greifst Du Medusa an, / während Phoebus Du in deiner Mitte wohnen lässt.)

Man tut dem Künstler wohl nicht allzu unrecht, wenn man die Übereinstimmung zwischen Text und Bild als lückenhaft bemängelt. Die Verse sagen aber unmissverständlich aus, worum es geht. Die Stadt verdankt ihren Reichtum (das Füllhorn) dem Getreidehandel. Danzig war in den Jahrzehnten um 1600 der wichtigste Ausfuhrhafen des für Westeuropa lebenswichtigen polnischen Getreides. In der Beschreibung, die Dickman seinem großen Stadtpanorama beigab, heißt es dazu: „Zuletzt lieber Leser, kan ich dich zu berichten keinen umbgang haben, dass die Stadt Dantzig von wegen der grossen menge des Korns gleichsam eine kleine Weltscheure sey, aus welcher Korn in alle andere Länder /---/ geführet wird ..." Danzig erhob aber auch den Anspruch, eine Stätte der Wissenschaft und gelehrter Studien zu sein, die die Barbarei (das Medusenhaupt) in ihren Mauern nicht duldete. Welche Bedeutung der Spiegel in diesem Zusammenhang hat, ist nicht ganz klar. Er kann eine Anspielung auf den Spiegel sein, den Perseus zu Hilfe nahm, um sich dem versteinernden Blick der Medusa nicht auszusetzen, er kann aber auch als Symbol der Prudentia, der Klugheit, den Bildungsanspruch der Stadt symbolisieren. Das ist hier vielleicht die wahrscheinlichere Deutung, fehlen im Stillleben denn sonst alle Anspielungen auf die Tugenden von Pallas und Apoll.

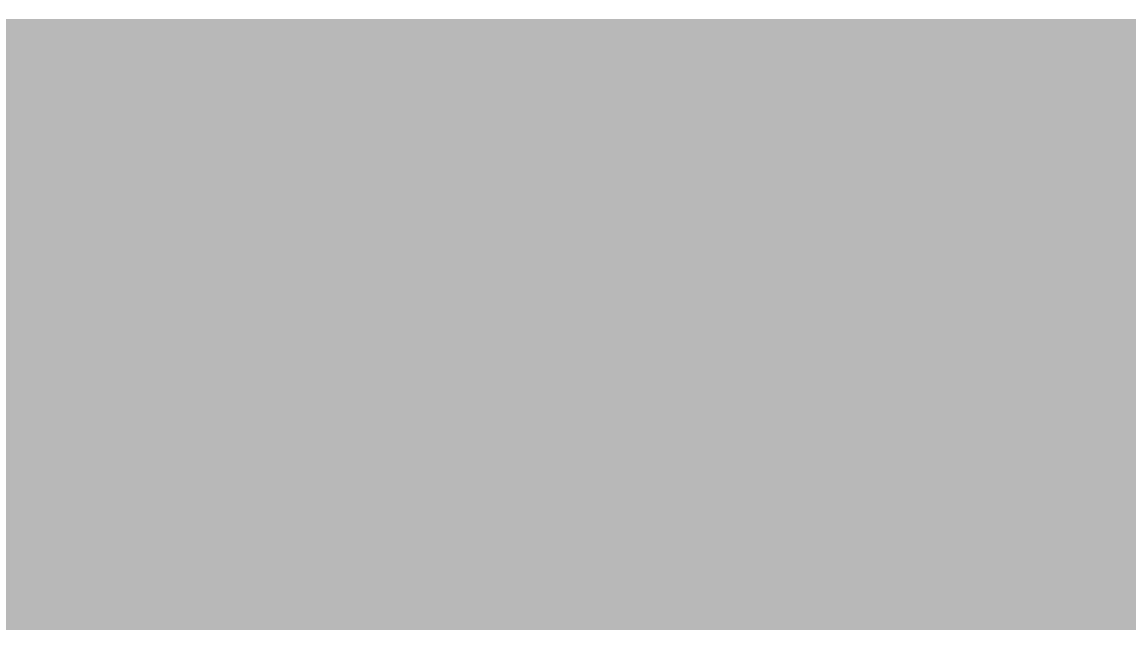

ABB. 5. DIE FESTUNG WEICHSELMÜNDE (NACH ÆGIDIUS DICKMANN UND DAS DANZIGER STADTBILD UM 1617).

Das erste Blatt der Folge nach dem Titelblatt stellt die Festung Weichselmünde dar (Abb. 5). Der runde Turm ragt in der Mitte der Komposition in die Höhe, die ihn umgebenden, sorgfältig ausgemauerten Bastionen strahlen Härte und Abwehrkraft aus, und die Wachtposten auf dem Wall und dem gezimmerten Bollwerk am Festungsgraben sind deutlich zu erkennen. Rechts im Bilde sehen wir, dicht gedrängt, Boote und zwei Segelschiffe. Links ist ein Zipfel der Ostsee zu erkennen, und im Hintergrund ahnt man die Türme von Danzig. Schon beim Aufschlagen der ersten Seite bekommt man einen Eindruck davon, wie wehrhaft die Stadt ist, während die Schiffe auf ihre Bedeutung als Handelshafen hinweisen.

Diesen Eindruck verstärkt das zweite Blatt der Folge, das den Mottlauhafen mit der Grünen Brücke zeigt (Abb. 6). Im Zentrum steht hier das Grüne Tor, eines der ersten und anspruchsvollsten Renaissancebauten der Stadt. Die Brücke, die die Rechtstadt mit der Speicherinsel verbindet, führt von unten links im Bilde auf das Tor zu. Fuhren, Lastträger und vornehm gekleidete Fußgänger zeugen vom lebhaften Treiben an diesem zentralen Ort des Hafens. Vor der Brücke liegen Segelschiffe, und ein mit Korn beladener Kahn gleitet 


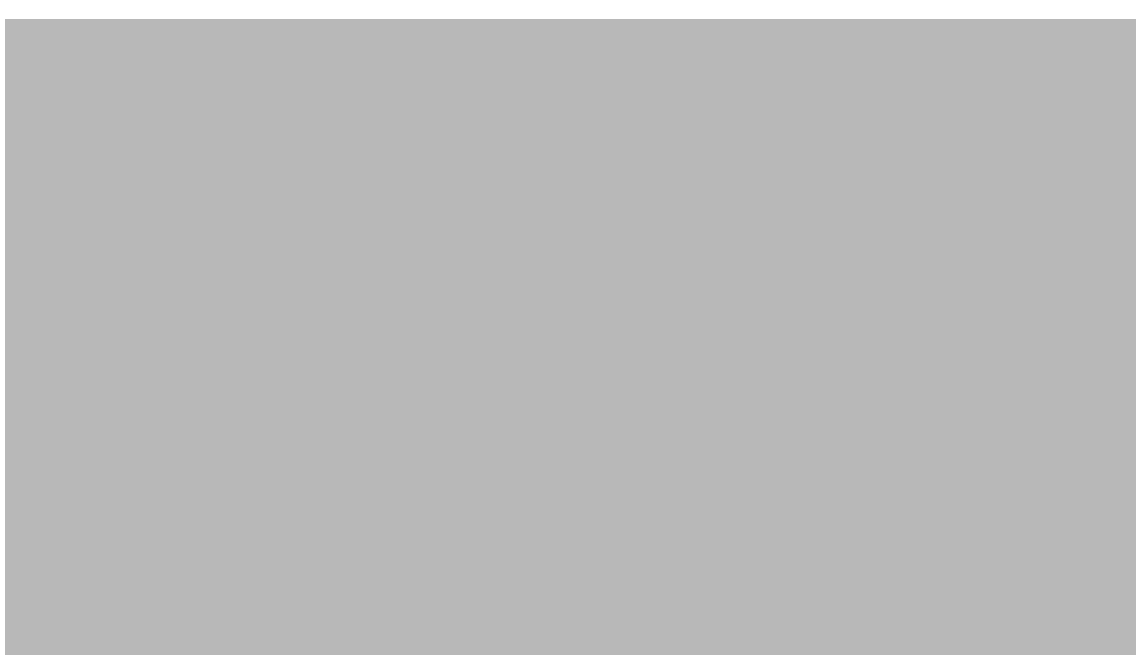

ABB. 6. MOTLAU UND GRÜNE BRÜCKE. FOTO: GNM.

mit umgelegtem Mast auf den Durchlass unter die Brücke zu. Das war sicher ein häufiger Anblick, der hier nun auch, wie absichtslos, auf den Getreidehandel als wichtigste Quelle des Danziger Reichtums hinweist.

Das Grüne Tor ragt hoch über die umgebenden Häuser hinaus und macht einen geradezu palastähnlichen Eindruck. Dickman hat die Proportionen des Gebäudes verändert und es viel höher dargestellt, als es in Wirklichkeit ist. Beträgt das Verhältnis zwischen Höhe und Breite des Gebäudes in der Realität etwa 2:3 ist es auf der Radierung fast 1:1. Ungeachtet solcher Abweichungen ist die Architektur auch im Detail sehr genau wiedergegeben. Die Zahl der Fenster und Öffnungen ist korrekt dargestellt und man erkennt sogar die verschiedenen Säulenordnungen an der Fassade. ${ }^{6}$ Bemerkenswert detailgetreu und fachgerecht ist auch die Konstruktion der Brücke abgebildet.

6 Dass das Tor heute vier, und nicht, wie auf der Radierung, drei Durchlässe hat, ist die Folge eines späteren Umbaus im Zusammenhang mit der Verlegung der Waage, die sich hier befunde hatte (und die Dickman auf der Radierung ausweist).

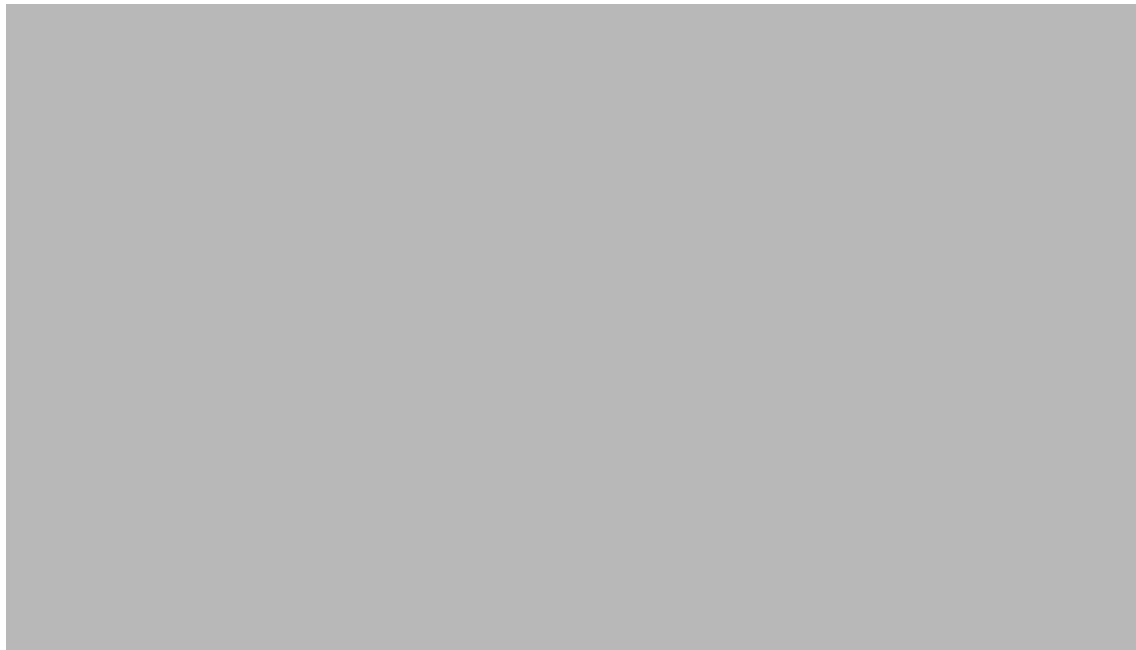

ABB. 7. DER LANGE MARKT MIT DEM RATHAUS (NACH ÆGIDIUS DICKMANN UND DAS DANZIGER STADTBILD UM 1617).

Durch das Grüne Tor gelangt man auf den Langen Markt, den vornehmsten Platz der Rechtstadt (Abb. 7). Dickmans Radierung zeigt den oberen Teil des Platzes und gibt auch den Blick frei in die Langgasse bis zum Langgasser Tor und dem Stockturm. Genau in der Mitte der Komposition steht das Rathaus, an der rechten Seite sind die damals gerade neu gestalteten Fassaden vom Artushof, dem Schöffenhaus und einigen Stadtpalästen sehr detailgetreu zu sehen. Interessant sind die vielen Verkaufsstände auch vor den vornehmsten Häusern. Wie auf allen anderen Ansichten der Folge ist die Höhe der Gebäude im Verhältnis zu ihrer Breite auch hier deutlich übertrieben.

Ein interessantes Detail ist der Brunnen, der mitten auf dem Platz steht. Dargestellt ist nicht der Neptunbrunnen, der heute an dieser Stelle seinen Platz hat. Diesen gab es 1617 noch gar nicht, er wurde erst 1633 errichtet. ${ }^{7}$ Seinen hohen repräsentativen Wert für die Stadt zeigt

7 Helmut Carl, „Der Neptunbrunnen auf dem Langen Markt zu Danzig“, Zeitschrift fü Kunstgeschichte, 6 (1937), 147-170; Lars Olof Larsson, „Studnia Neptuna na Diugim Targu w
Gdańsku (Der Neptunbrunnen auf dem Langen Markt in Danzig)“, Porta aurea, 10 (2011), 5-23. 
sich unter Anderem darin, dass Curicke ihm in seiner Beschreibung der Stadt von 1687 sogar eine eigene Abbildung widmet. Vielleich hat Dickman den Brunnen abgebildet, der vorher hier stand. Über ihn ist wenig bekannt. 1549 sind Zahlungen für Rohre und andere, nicht spezifizierte Arbeiten eines "Snitzer(s) vor tyn“ (Zinnschneider) dokumentiert, die sich auf den „Borne, vor dem howe“ (den Brunnen vor dem Hofe, d.h. dem Artushof) beziehen. ${ }^{8} \mathrm{Ob}$ es sich bei der Arbeit des "Zinnschneiders" um Zierrat aus Zinn, wie Carl vermutet, oder vielleicht doch eher um Rohre handelte (die wohl nicht aus Zinn, sondern aus Blei waren), ist aus den Eintragungen leider nicht zu ersehen. Wir wissen natürlich auch nicht, ob diese Arbeiten in Zusammenhang mit der Errichtung eines neuen Brunnens standen oder ob es sich um Ausbesserungsarbeiten an einem bestehenden Brunnen handelte. Bisher wurde meist angenommen, dass es sich bei diesem um einen eher bescheidenen, einfachen "Röhrkasten“ handelte, eine Abbildung von einem solchen ist aber nicht bekannt. ${ }^{9}$ Dass auf der großen Vogelschauansicht der Stadt von etwa 1600 an dieser Stelle kein Brunnen zu sehen ist, bedeutet nicht unbedingt, dass es einen solchen damals nicht gab. Um der größeren Klarheit willen sind an den Straßen keine der zahlreichen Vorbauten und Ziehbrunnen dargestellt worden, von denen es nachweislich viele gab (Abb. 11). Wenn nun Dickmans Radierung den alten Brunnen zeigt, war dieser durchaus sehr repräsentativ und kunstvoll gestaltet. Wir sehen da einen eleganten sogenannten Kandelaberbrunnen mit kreisrundem und nicht besonders breitem Brunnenbecken. Der Brunnenpfeiler wird durch zwei flache Schalen unterteilt und oben von einer nicht genau zu erkennenden Figur oder Vase gekrönt, von der Wasserstrahlen in einem eleganten Bogen in die obere Schale fallen. Auf der unteren Schale sitzen Figuren, die, vermutlich aus Krügen, dünne Wasserstrahlen in das Becken gießen. Um das überfließende Wasser abzuleiten, ist der Boden unter dem Brunnen und bis zum Rinnstein glatt gepflastert. Ähnliche Wasserkünste finden sich in großer Zahl in der Graphik und in den Gemälden von Jan Vredeman de Vries. Wenn wir aber annehmen wollen, dass der Danziger Brunnen schon um 1549 oder davor entstanden ist, kommen Anregungen von Vredeman de Vries natürlich nicht infrage. Der

8 Carl, ,Der Neptunbrunnen auf dem Langen Markt zu Danzig“, 162 (Urkundenanhang).

9 Ibidem, 148
Kandelabertypus war aber keine Erfindung von ihm, den gab es viel früher, vor allem in Italien und in Frankreich. Es wäre also durchaus denkbar, dass ein Brunnen, wie ihn Dickman abbildet, schon um die Mitte des Jahrhunderts in Danzig geschaffen wurde. Nun wissen wir, dass der Rat schon um 1605 den Entschluss fasste, den bestehenden Brunnen zu ersetzen. Die Gründe dafür kennen wir nicht; vielleicht gab es, wie so oft, technische Probleme mit den Wasserrohren, die es nahelegten, einen ganz neuen Brunnen anzulegen. Vielleicht machten sich aber auch gewachsene repräsentative Ansprüche geltend. ${ }^{10} \mathrm{Ab} 1606$ fertigte der Bildhauer Abraham van den Blocke die Steinelemente eines neuen Brunnens, und Anfang 1615 vollendete der eigens für die Bronzeplastiken nach Danzig berufene Peter Husen den großen Neptun. ${ }^{11}$ Dass die Errichtung des Brunnens sich dann bis 1633 hinziehen sollte, war damals sicher nicht vorhersehbar. Vor diesem Hintergrund fragt man sich, warum Dickman den neuen, viel prächtigeren Brunnen nicht abbildete, dessen Aufstellung 1617 nach allem zu urteilen unmittelbar bevorstand. Wenn er in den Jahren vor der Veröffentlichung seiner Drucke in Danzig lebte, was anzunehmen ist, kann ihm ja kaum entgangen sein, dass ein neuer Brunnen in Arbeit war, und er dürfte auch die beteiligten Künstler gekannt und die fertigen Teile gesehen haben. Schon 1609 hat Isaac van den Blocke in seinem großen Deckengemälde der Sommerratsstube einen Brunnen vor dem Artushof dargestellt, der mit dem damals noch nicht fertigen Neptunbrunnen sehr gut übereinstimmt. Dass der um Realitätstreue sonst stets bemühte Dickman in dieser Situation anstelle des noch vorhandenen Brunnens einen solchen nach seiner eigenen freien Erfindung vor dem Artushof dargestellt hätte, ist schwer vorstellbar aber natürlich nicht auszuschließen. Restlos wird sich diese Frage wohl nicht klären lassen.

Das nächste Blatt zeigt das Ensemble um den Stockturm mit der Peinkammer und der Stadtseite des Hohen Tores (Abb. 8). Der Stockturm steht genau in der Mitte der Komposition und ragt, deutlich

10 Es sei an die gleichzeitige Neugestaltung wichtiger Räume des Rathauses und an den Umbau der Fassade des Artushofes erinnert.

11 Es ist nicht bekannt, von wo Peter Husen nach Danzig kam. Wahrscheinlich kam er aus Augsburg, er kann aber auch aus den Niederlanden berufen worden sein. Carl, „Der Neptunbrunne auf dem Langen Markt zu Danzig“, 150 und Larsson, „Studnia Neptuna na Dugim Targu w Gdańsku“, 14f. Zu Husen siehe auch Lars Olof Larsson, „Bemerkungen zur Bildhauerkunst am dänischen Hofe im 16. Und 17. Jahrhundert", (zuerst in Munchener Jahrbuch der bildenden Kunst, 26 (1975)) Wege nach Süden. Wege nach Norden. Aufsätze zu Kunst und Architektur (Kiel: Verlag Ludwig, 1998), 117-135, vor allem 130-132. 


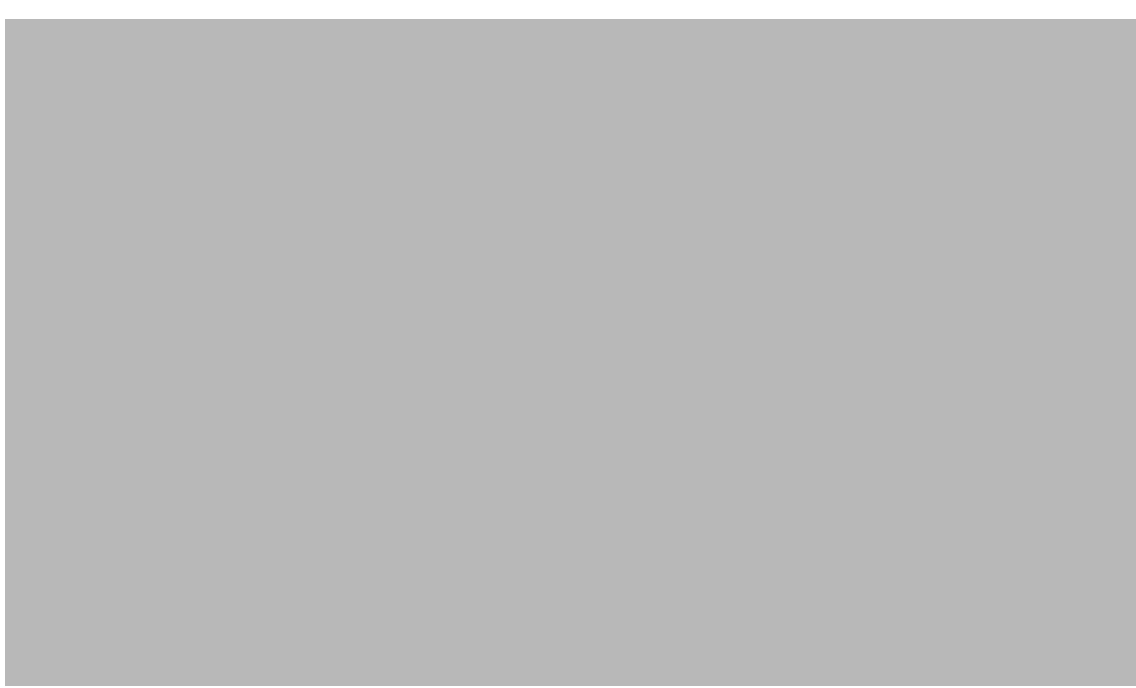

ABB. 8. STOCKTURM UND HOHES TOR (NACH ÆGIDIUS DICKMANN UND DAS DANZIGER STADTBILD UM 1617)

überlängt, in die Höhe. Rechts blicken wir auf den Dominickplan, der zur Stadt hin vom Langasser Tor, der Georgshalle und dem Zeughaus begrenzt wird. Im Hintergrund sind die Türme der Altstädter Kirchen St. Katharinen und St. Bartholomäi und des Altstädter Rathauses zu sehen. Die Staffagefiguren, Pferdefuhren und Fußgänger, nehmen Bezug auf das Hohe Tor als die Hauptzufahrt in die Stadt; Anspielungen auf die Funktion von Stockturm und Peinkammer sind nicht zu erkennen. Wie Curicke in seiner Beschreibung der Stadt berichtet, wurden im Stockturm Verbrecher gefangen gehalten, die "so etwan auf den Halß sitzen, oder auch solche Schelmstücke begangen, dass die deßwegen in des Henckers Hände gerathen", und in der Peinkammer wurden „Übelthäter examiniert“. Vor dem Turm, zum Domnickplan hin, stand seit 1604 der Pranger. ${ }^{12}$ Auf der Abbildung des Stockturmes in Curickes Stadtbeschreibung wird gezeigt, wie ein Gefangener auf einem erhöhten Podium am Turm (das auch auf Dickmans Radierung zu sehen ist) unter Zulauf einer Rosenberg (Hamburg: Danziger Verl.-Ges. Paul Rosenberg, 1979), $59 \mathrm{f}$

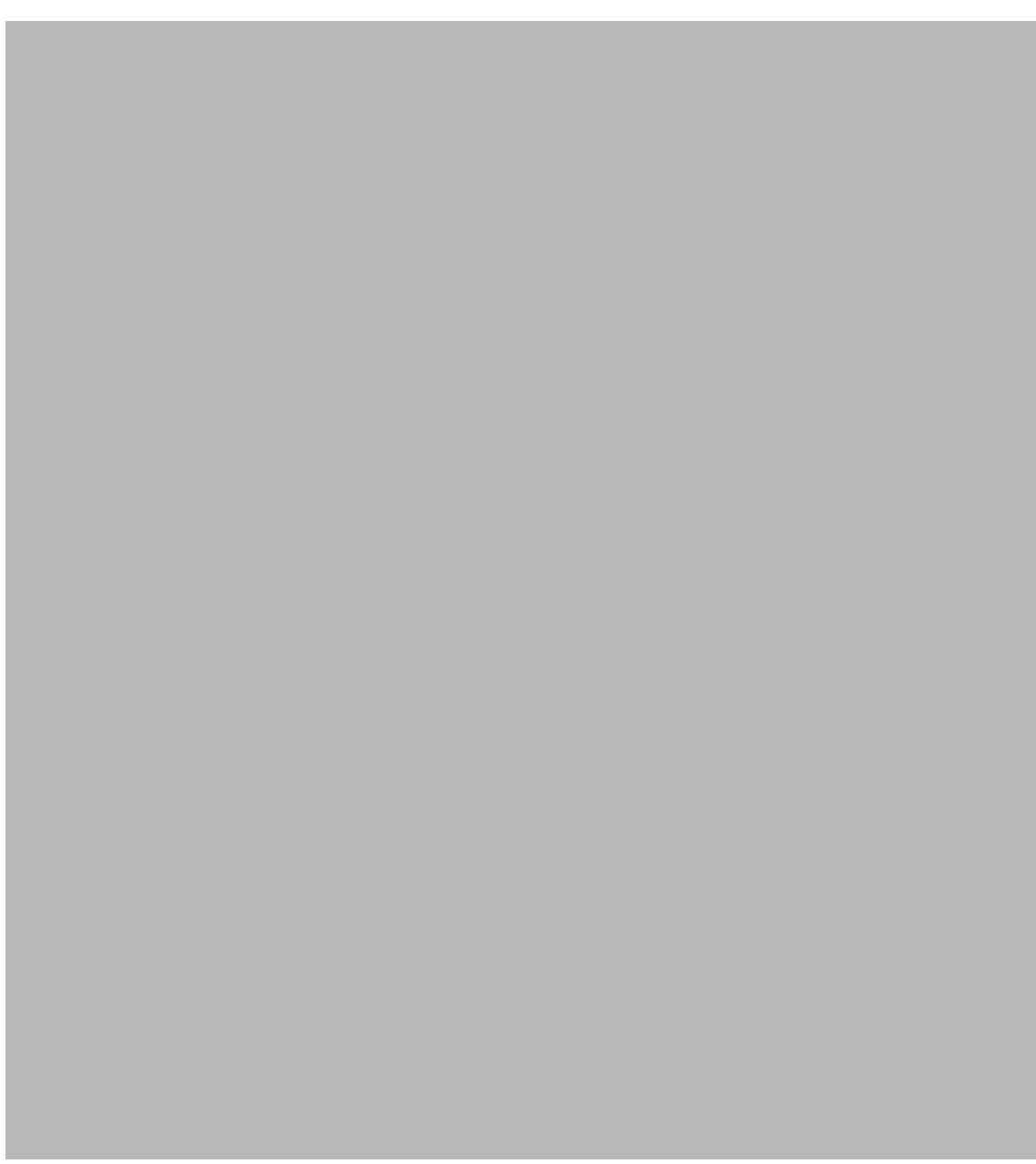

ABB. 9. DER STOCKKTURM. AUS: CURICKE, DER STADT DANTZIG HISTORISCHE BESCHREIBUNG $(1687)$

großen Menschenmenge ausgepeitscht wird (Abb. 9). Dickman, de den Staffagefiguren auf seinen Drucken meist viel Aufmerksamkeit widmet und sie sehr informativ darstellt, wollte offenbar seinem Publikum den Anblick solcher Szenen ersparen.

Es folgt die Ansicht des Holzmarktes, eines großen offenen Platzes, der sich als Fortsetzung des Dominickplans zur Altstadt hin erstreckte 


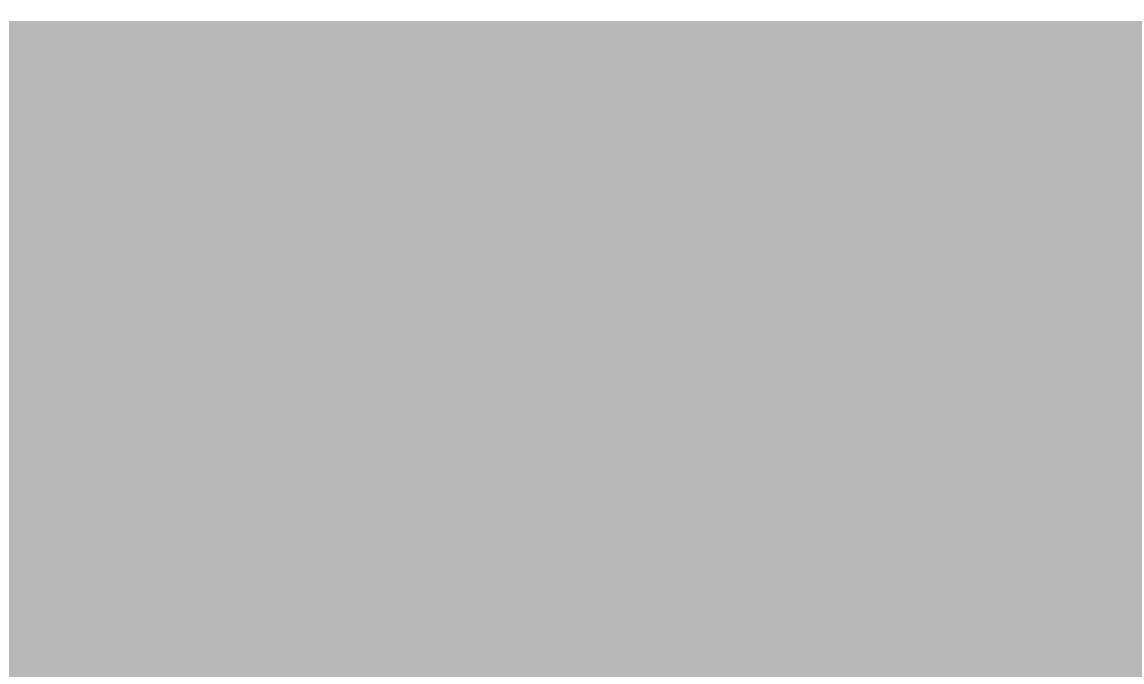

ABB. 10. DER HOLZMARKT. FOTO: GNM.

(Abb. 10). Wir blicken in Richtung Osten, die Häuserfronten der Altstadt bilden den linken Rand des Platzes. Rechts sehen wir Teile des älteren Stadtbefestigungsgürtels und hohe, schlanke Türme, die zur Trennmauer zwischen Altstadt und Rechtstadt gehören. Sie bilden auch auf diesem Blatt den Blickfang. Größeres Interesse beansprucht hier aber die Darstellung des regen, geschäftigen Treibens auf dem Platz. Mit Holz beladene Fuhren kommen an oder warten schon darauf, entladen zu werden, leere Wagen stehen herum, und zwischen ihnen bewegen sich Kaufleute und Männer mit Beilen und anderem Werkzeug, die sie als Holzfäller oder Zimmerleute ausweisen.

Soweit könnten die Radierungen als Anleitung eines virtuellen Gangs durch die Stadt gesehen werden. Mit Hilfe einer sehr detaillierten und um 1600 zu datierenden Darstellung der Stadt aus der Vogelperspektive lässt sich das gut veranschaulichen (Abb.11). ${ }^{13}$

13 Die Zeichnung (schwarze Tinte und Sepia) befindet sich im Krigsarkivet in Stockholm (Utländska stads- och fästningsplaner, Tyskland, Danzig, nr. 13). Sie misst 100×73 cm. Heinz Lingenberg schreibt sie Anton Möller zu und datiert sie überzeugend um 1600 (Heinz Lingenberg, „Der älteste Grundrissplan Danzig - ein Werk Anton Möllers?", Unser Danzig, 1-2 (1984) und Ulla Ehrensvärd, Pellervo Kokkonen, Juha Nurminen, Mare Balticum. 2000 Jahre Geschicht der Ostsee (Helsinki: Verlags-Ag. Otava; John Nurminen-Stiftung, 1996), $100 \mathrm{f}$. ABB. 11. ANTON MÖLLER (?), DANZIG AUS DER FOGELPERSPEKTIVE, UM 1600. FOTO:
KRIGSARKIVET, STOCKHOLM. 
Der Besucher gelangt an der Festung Weichselmünde vorbei in den Hafen und legt an der Grünen Brücke an. Von dort geht er über den Langen Markt, am Rathaus vorbei und gelangt durch die Langgasse zum Stockturm und zum Dominickplan am westlichen Ende der Stadt. Weiter geht es dann zum Holzmarkt. Dass es keinesfalls anachronistisch wäre, die Ansichten so zu betrachten, beweist z. B. der Werbetext auf einem der beiden Titelblätter von Claus Jansz. Visschers Folge Plaisante plaetsen, einer Sammlung von Radierungen mit Motiven aus der Umgebung von Haarlem, wo es heißt:

Plaisante plaetsen hier, meught ghij aenschouwen radt.

Liefhebbers die geen tijt en hebt om veer te reijsen,

Gheleghen buijten de ghenoechelijke Stadt,

Haerlem of daeromtrent, koopt zonder lang te peijsen!

(Hier könnt Ihr angenehme Plätze schnell anschauen / Liebhaber die keine Zeit habt, weit zu reisen / gelegen vor der gemütlichen Stadt / Haarlem oder in der Nähe. Kauft ohne lang zu feilschen!).

Dem, der keine Zeit zu Verreisen hat, bieten sich die Ansichten als Ersatz an, gewissermaßen für eine Reise in der Phantasie. ${ }^{14}$ Die Radierungen der Folge zeigen Orte am Rande oder außerhalb Haarlems, die zu einem Ausflug einladen. Wenn Visscher sie plaisante plaetsen nennt, überträgt er den humanistischen Topos des locus amoenus in die niederländische Gegenwart.

Mit der Darstellung des Holzmarktes verlässt die Reihenfolge der Blätter freilich die angedeutete Systematik. Es folgt jetzt die Ansicht des Motttlauhafens am Krantor (Abb. 12). Dieses Wahrzeichen der Stadt ist allerdings hinter den vielen Schiffsmasten kaum zu erkennen. Links sehen wir das Nordende der Speicherinsel und dahinter wiederum eine Menge Schiffsmasten. Eine Fähre mit einem Ruderer und stehenden Passagieren belebt den Vordergrund. Das Blatt vermittelt einen besonders anschaulichen Eindruck vom lebhaften Betrieb im Hafen. Man hätte nun erwarten können, dass die Ansicht der Neuen Mottlau, also des ausgebaggerten Mottlauarms östlich der Speicherinsel mit ihren großen Speichern, als nächstes

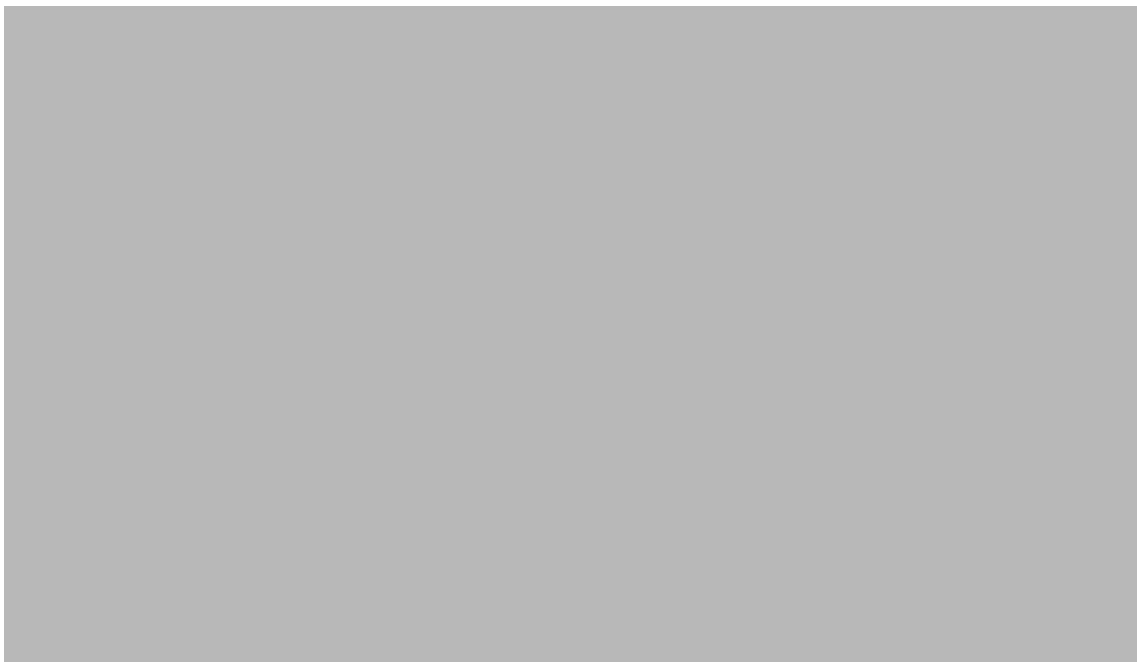

ABB. 12. DER MOTLAUHAFEN MIT DEM KRANTOR. FOTO: GNM.

Blatt die Darstellung von Hafen und Speicherinsel ergänzen würde. Stattdessen folgt aber erst eine Ansicht vom Dominickplan, vom Norden aus betrachtet. Im Hintergrund sehen wir den hohen Stockturm und an der linken Seite die rückwärtige Fassade des Zeughauses. Der Platz ist von Fuhren und Wagen auf dem Weg aus der Stadt durch das Hohe Tor hinaus, von abgestellten Pferdewagen und von Fußgängern und spielenden Kindern belebt. Der Pranger, der vor dem Stockturm stand, ist nicht zu sehen. Auch in diesem Fall ist Curicke expliziter, auf seiner Abbildung des Platzes sehen wir nicht nur den Pranger, sondern auch, wie ein Sträfling auf das Holzpferd gesetzt worden ist.

Die folgenden Blätter zeigen die Große Mühle und ein Stück der Langgasse. Die große Mühle lag auf einer Insel des Radaunekanals in der Altstadt (Abb. 13). Sie stammte aus der Ordenszeit und blieb bis zur Zerstörung 1945 in Betrieb. Das monumentale Gebäude ist von Osten her abgebildet; am Giebel sehen wir den großen Backofen mit seinem hohen Schornstein. Vor dem Gebäude kommt eine schwer beladene Fuhre mit Kornsäcken an; auf der Straße entlang des Kanals entfernt sich ein Mann mit zwei Eseln, auf deren Rücken er Mehlsäcke geladen hat. Auch hier lässt Dickmann das Gebäude 


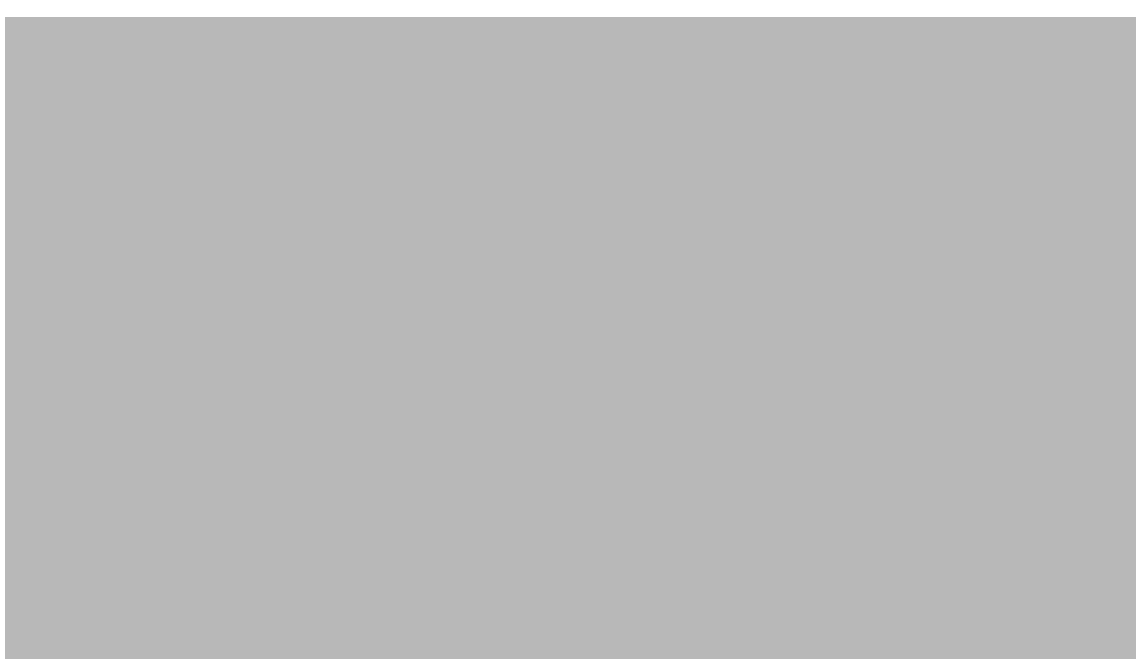

ABB. 13. DIE GROSSE MÜHLE. FOTO: GNM.

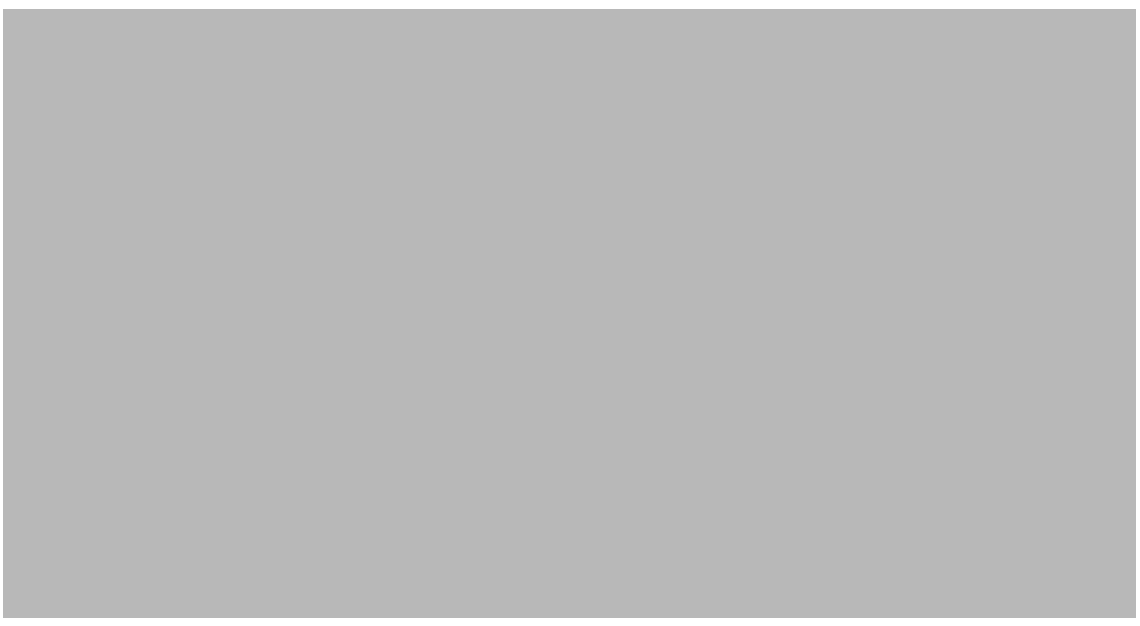

ABB. 14. DIE LANGGASSE. FOTO: GNM.

größer erscheinen, als es in Wirklichkeit war; mit seinem auf sieben Geschosse verteilten Mahlbetrieb hat es aber die umgebende, eher bescheidene Bebauung sicher auch in Wirklichkeit wesentlich überragt.

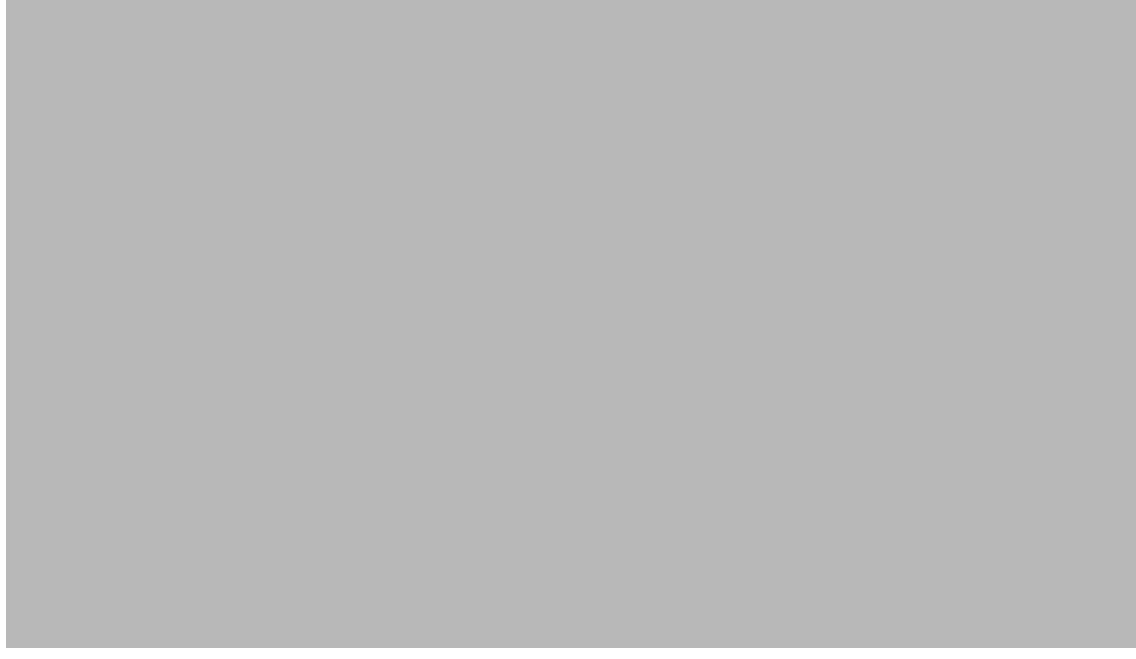

ABB. 15. DAS HOHE TOR. FOTO: GNM.

Die Darstellung der Langgasse ist strikt zentralperspektivisch komponiert mit dem Langgasser Tor und dem Stockturm dahinter als Fluchtpunkt (Abb. 14). Ein eleganter, gedeckter Wagen, reitende Kavaliere, ein Jäger mit Jagdbeute und Windhunden und vornehm gekleidete Menschen zeigen, dass diese Straße, neben dem Langmarkt, die vornehmste Wohnadresse der Stadt war. Dickman hat sie dementsprechend auch bedeutend breiter dargestellt, als sie in Wirklichkeit ist. Die Häuser zeigen alle würdige Fassaden, anders als auf der Ansicht des Langmarktes sind aber einzelne Wohnhäuser nicht besonders hervorgehoben.

Die letzten zwei Ansichten der Folge zeigen die beiden wichtigsten Stadttore, das Leichnamstor im Norden und das Hohe Tor (Abb. 15) im Westen der Stadt. Beide Blätter lassen eher an Landschaftsdarstellungen als an Stadtveduten denken, die Architektur spielt hier eine nur untergeordnete Rolle. Das spätmittelalterliche Hl. Leichnamstor erscheint am äußersten Bildrand links, ihm vorgelagert liegt ein hohes, modernes Erdwerk, an dem entlang die Zufahrtstraße verläuft. Rechts am Rande sieht man die Hl. Leichnamskirche. Von dem dazu gehörenden großen Hospital ist nichts zu sehen. Im Hintergrund 
sehen wir den Hagelsberg, an dem die Spuren des durch den Bau der Wälle intensiven Kiesabbaus deutlich zu erkennen sind.

Sehen wir vom letzten Blatt ab, das einen Plan der Stadt zeigt, wird die Folge der Ansichten durch das Hohe Tor mit dem weitläufigen Gelände davor beendet. Das entspricht der Logik, die wir für die ersten sechs Blätter angenommen haben: Bei einem imaginären Besuch der Stadt kommen die Besucher von der Seeseite an und betreten die Stadt durch das Grüne Tor, das wichtigste Tor zum Hafen. Nach beendetem Besuch verlassen sie die Stadt durch das Hohe Tor, das wichtigste Tor an der Landseite, und blicken dann auf die Stadt und ihre eindrucksvollen Befestigungen zurück. Auf der Landstraße herrscht reger Verkehr, und der Rossmarkt und die Pferdeschwemme am Radaunekanal sind lebhaft frequentiert. An der Steinbrücke über den Kanal steht das hohe Gebäude der Lohmühle, und im Hintergrund erkennt man den Bischofsberg.

Der Stadtplan schließlich, der die Folge beschließt, bildet die analytische Ergänzung zum Stadtpanorama des Titelblattes, mit dem sie eingeleitet wurde. Es handelt sich um einen der ältesten Grundrisse Danzigs. Wenn auch nicht maßstabsgerecht gezeichnet, gibt er eine anschauliche Vorstellung von der Struktur der Stadt mit ihrer Einteilung in Altstadt, Rechtstadt und Neustadt und von der landschaftlichen Situation mit den Flüssen Mottlau und Weichsel bis hin zur Ostsee.

Vergleichen wir Dickmans Ansichten z. B. mit den Abbildungen in Curickes einige Jahrzehnte jüngerer Beschreibung der Stadt, fällt auf, dass Dickman nie einzelne Gebäude isoliert darstellt. Auch Repräsentationsbauten wie die Rathäuser, der Artushof, das Grüne Tor oder das Zeughaus werden als Teile von ganzen Milieus gezeigt. Bemerkenswert ist es auch, dass die Kirchen ganz fehlen. Es fehlt auch das Gymnasium, das seit 1558 im ehemaligen Franziskanerkloster in der Vorstadt untergebracht war. ${ }^{15}$ Das überrascht umso mehr als auf dem Titelblatt die Pflege von Kunst und Wissenschaft so stark hervorgehoben wird, und Dickman das Gymnasium in der Beschreibung der Stadt, die er dem großen Stadtpanorama beigefügt hat, rühmt. Als einziges einzelnes Gebäude beherrscht nur die große Mühle eine Ansicht. Dazu passt, dass eine Mehrzahl der Ansichten dem wirtschaftlichen Herzstück der Stadt, dem Hafen und der Speicherinsel sowie dem für Handel und Gewerbe wichtigen Dominikplan und dem Holzmarkt gewidmet sind. Dazu passt auch dass die Legende auf dem Blatt mit der Darstellung der Grünen Brücke und des Grünen Tors lediglich auf die in einem Teil von diesem Gebäude untergebrachte Waage hinweist, nicht jedoch auf das prachtvolle Gebäude als solches. Vor diesem Hintergrund hätte man nun vielleicht auch erwarten dürfen, dass auf dem Blatt mit dem Dominickplan der wichtige Dominick-Jahrmarkt dargestellt worden wäre. Das ist aber nicht der Fall. Ungeachtet solcher Auslassungen und anders als der lateinische Titel der Folge nahelegt, ging es Dickman offensichtlich vor allem darum, die wirtschaftliche Prosperität und das Leben und Treiben in der Stadt - und nicht so sehr die einzelnen Gebäude - zu zeigen. Vielleicht gibt das auch einen Fingerzeig, an welche Zielgruppen er sich in erster Linie wandte. Vermutete er seine Käufer eher unter den Kaufleuten, die mit Danzig Handel trieben als unter eher bildungsorientierten Schichten, die sich für das ferne Danzig vielleicht ohnehin kaum interessierten?

Dickmans Ansichten von Danzig gelten zu Recht als eine kulturhistorisch hervorragende Quelle, ihr künstlerischer Wert wird aber meist als gering geschätzt. Rudolf Bergaus Urteil von 1865, dass die „Ansichten /---/ künstlerisch ohne Bedeutung aber in antiquarischer Hinsicht /---/ von großem Interesse“ seien, ist nie ernsthaft widersprochen worden. ${ }^{16}$ Damit wird man Dickmans Leistung aber nicht gerecht. Vergleicht man seine Blätter mit Arbeiten von Künstlern wie z. B. Claes Jansz. Visscher oder Jan van de Velde, erkennt man zwar, dass ihnen die Eleganz in der Strichführung oder die atmosphärische Wirkung, die diese Künstler zu erzielen vermochten, fehlen. Im Vergleich mit den beiden Niederländern fällt auch seine recht schulmäßige Anwendung der Zentralperspektive auf. Unsere Anerkennung verdient dagegen Dickmans Fähigkeit, die einzelnen Motive in der Gesamtheit der Darstellung aufgehen zu lassen, ungeachtet der Detailtreue und Präzision ihrer Wiedergabe. Die Genauigkeit, mit der er die Formen der Architektur, die Bauweise von Brücken, Zäunen und anderen scheinbaren Nebensächlichkeiten sowie auch alle Einzelheiten der Staffage (z. B. die verschiedenen Wagentypen) wiedergibt, begründen nicht nur den kulturhistorischen 
Wert der Darstellungen, sie stellen auch einen wichtigen Aspekt ihrer künstlerischen Eigenart dar.

Zur historischen Bedeutung der vierzehn Ansichten von Danzig trägt schließlich ihre sehr frühe Entstehung bei. Vergleichbare Darstellungen von Plätzen und Gebäuden gab es damals vor allem von Rom und wenigen anderen italienischen Städten, wobei das Interesse dort hauptsächlich den Ruinen der antiken Bauwerke galt. Unter den west- und nordeuropäischen Städten findet sich Vergleichbares nur in der Handelsmetropole Amsterdam. Dort sind um 1600 vereinzelt druckgraphische Darstellungen von Gebäuden und Plätzen entstanden. Hier übten der Hafen, der lebhafte Handel und die Fülle von Waren und Menschen aus aller Herren Länder offenbar eine Faszination aus, die es lohnend erscheinen ließ, graphische Darstellungen von den kommerziellen Brennpunkten der Stadt zu produzieren. Eine wichtige Voraussetzung war dabei natürlich auch das Vorhandensein eines leistungsfähigen Graphik- und Kunstmarktes. Beispielhaft für die seit Mitte des 16 Jahrhunderts gewachsene, europaweite kommerzielle Dominanz der niederländischen Druckgraphik stand zunächst die Antwerpener Offizin "Aux quattre vents" von Hieronymus Cock. Cocks Verlagsprogramm war breit angelegt; sehr wichtig waren seine vielen Ansichten von römischen Ruinen. Unter dem Druck der politischen Entwicklung verlagerte sich um 1600 der Schwerpunkt des Graphikhandels und der Produktion von Druckgraphik nach Amsterdam. Hier und in angrenzenden Städten, wie Haarlem, entwickelte eine kleine Gruppe junger Künstler auch neuartige Formen von Landschaftsdarstellungen, kleinformatige Radierungen von scheinbar anspruchslosen, meist ländlichen Motiven, zu denen sich auch Architekturdarstellungen und einheimische städtische Motive gesellten. Für unseren Zusammenhang am wichtigsten war Claes Jansz. Visscher, der seine Laufbahn als Künstler begann, sich aber bald vor allem dem Verlagsgeschäft widmete. ${ }^{17}$ Zwischen ihm und Dickman bestand, wie wir gesehen haben, um 1625 eine Verbindung, ohne dass wir sagen können, ob sie auch persönlicher Natur war. Dass Dickman Publikationen von Visscher kannte, dürfte aber als sicher gelten. Ein Indiz dafür ist die Ähnlichkeit

17 Zu Visscher siehe Maria Simon, Claes Jansz. Visscher (unpublizierte Diss. Freiburg i. Br., 1958) und Hollstein's Dutch and Flemish Etchings, Engravings and Woodcuts c. 1450-1700, vol. 38-39 (1991) des Titelblattes seiner Danziger Ansichten mit dem Titelblatt der Folge Plaisante plaetsen, die Visscher 1611 herausgab (Abb. 2). Hier blickt man, wie auf dem Titelblatt Dickmans, durch einen weiten Bogen auf eine dahinter liegende Landschaft, die wie auf einer Bühne präsentiert wird. Dickman kann auch die Anregung, Plätze und Straßen seiner Stadt in Radierungen darzustellen und diese als zusammenhängende Folge zu publizieren, von Visschers 1611 datierten Ansichten von Amsterdam und von der schon genannten Folge Plaisante plaetsen bekommen haben..$^{18}$ Visschers Folgen sind von etwa gleichem Umfang, nur sind Dickmans Drucke etwas größer als Visschers, und sie zeigen, wie wir gesehen haben, ausschließlich städtische Motive.

Dass eine solche Folge topographischer Ansichten gerade in Danzig geschaffen wurde, sollte vor dem Hintergrund der vielen repräsentativen privaten und öffentlichen Bauprojekte betrachtet werden, die Danzig während der Jahrzehnte um 1600 zur prachtvollsten Stadt im gesamten Ostseeraum machte. Der Lokalstolz, der aus dem Titel von Dickmans großem Stadtpanorama spricht, drückt vermutlich ein in der Stadt verbreitetes Gefühl aus: DANTISCUM SEPTENTRIONIS, OCCIDENTIS ET TOTIUS ORBIS HODIE EMPORIUM FREQUENTISSIMUM (Danzig, die heute meist besuchte Handelsstadt des Nordens, des Westens und der ganzen Welt). Dass Danzig es verdiente, der Welt in einer Bilderfolge vorgestellt zu werden, konnte keinem Zweifel unterliegen! Auch in Amsterdam teilte man offensichtlich diese Auffassung. Dass Visschers sie in sein Verlagsprogramm aufnahm, darf als Beweis dafür gelten. Man konnte offenbar damit rechnen, dass diese Blätter in Amsterdam auf Interesse stoßen würden. Dieses Interesse verdankte Danzig seiner wirtschaftlichen Bedeutung. Die Stadt war nicht nur der wichtigste Ausfuhrhafen für polnisches Getreide, sie war Anfang des 17. Jahrhunderts überhaupt der mit Abstand bedeutendste Handelshafen des ganzen Ostseeraumes, und ein großer Teil des Handels wurde mit niederländischen Schiffen abgewickelt.

Vor diesem Hintergrund wüsste man gern etwas mehr über den Künstler. Außer den hier vorgestellten Stadtansichten und

18 Hollstein's German Etchings, Engravings and Woodcuts c. 1400-1700, Nr. 144-147; Simon, Claes Jansz. Visscher, Nr. 147-150. Visscher bildet den Damm, die Börse, die Fleischhallen und den Fischmarkt ab. Diese Darstellungen sind auch zusammen mit einer Gesamtansicht de Stadt vom Hafon aus gesehen und mit einer langen Beschreibung auf einem Bogen montiert übrliefert (Hollstein's German Etchings, Engravings and Woodcuts c. 1400-1700, Nr. 147a). 
dem großen Stadtpanorama, die beide im selben Jahr entstanden sind, ist nur ein einziges Werk von seiner Hand bekannt, eine auf 1618 datierte Radierung, die den „Besuch der Bauern bei dem Rechtsanwalt" darstellt. Es ist eine humoristische, um nicht zu sagen burleske Genreszene im holländischem Stil. Dem Blatt hinzugefügt sind recht holprige, satirische Verse in deutscher Sprache. Die ungewöhnliche Schreibweise schul. für sculpsit in der Signatur mach einen überraschend unprofessionellen Eindruck. ${ }^{19}$ War Dickman als Radierer vielleicht ein begabter Laie und eigentlich in einem anderen Beruf zu Hause? Von seinem Leben wissen wir nur, dass er nach eigenem Bekunden aus Danzig stammte, und dass er sich 1624 in Haarlem aufhielt. In diesem Jahr wurde ihm von der Stadt Danzig über einen Mittelsmann eine Anstellung als Ingenieur in Aussicht gestellt. ${ }^{20} \mathrm{Ob}$ ihn das Angebot erreichte und ob er es annahm, ist nicht bekannt. Vielleicht dürfen wir aber aus dem Angebot schließen, dass er eigentlich im Baugewerbe tätig war. Für eine solche Vermutung lassen sich in den Radierungen, wie wir gesehen haben, schließlich auch Indizien finden. Wie dem auch sei, Dickmans Ansichten von Danzig verdienen in dokumentarischer wie in künstlerischer Hinsicht unsere Aufmerksamkeit als die zweifellos bedeutendsten topographischen Darstellungen in Nordeuropa vor Erik Dahlbergs Svecia antiqua et hodierna.

Lars Olof Larsson: "The Most Noble Squares and Buildings to Be Seen in Danzig": Aegidius Dickman's Views of Danzig IN 1617

KEYWORDS: EARLY MODERN PRINTED TOPOGRAPHICAL VIEWS; PRINT PROduction in the Netherlands; topography OF DanZig/GDÀnSK

\section{SUMMARY}

The paper discusses a set of etchings depicting different buildings in Gdansk (Danzig) and different parts of the city, first published in 1617. The artist was the little known Ægidius Dickman, active in Gdánsk and probably also in the Netherlands in the first quarter of the 17th century. In the same year that these etchings were published, Dickman also finished a large birds-eye view of Gdánsk. The set of town views and the panorama were both republished by Claes Janszoon Visscher in 1625

The author of the article discusses the relationship between Dickman and Visscher and their collaboration on this project, as well as their wider artistic relationship. Dickman seems to have been trained in the Netherlands, the etchings proving his familiarity with Visscher's topographical prints.

\section{CV}

Lars Olof Larsson is Professor Emeritus at the University of Kiel. Has acted as visiting professor at the Universities of Boston and Munich and at the Estonian Art Academy in Tallinn. Fellowships at Harvard Center for Renaissance Studies, Villa "I Tatti" in Florence and at the Netherlands Institute for Advances Study (NIAS), in Wassenaar. Doctor honoris causa at the Estonian Academy of Art, Tallinn. For several years chairman of the CMA Böckler-Mare Balticum-Stiftung.

Main fields of research: European sculpture and court art, $16^{\text {th }}-18^{\text {th }}$ centuries, Scandinavian art of the $19^{\text {th }}$ and early $20^{\text {th }}$ centuries, German and Scandinavian architecture of the $20^{\text {th }}$ century.

Recent works include a monograph on rhetorical aspects of Renaissance and Baroque portraiture, and articles on the Flemish and Dutch impact on Early modern architecture in Northern Europe. 
